\title{
Op art et roman virtuel : correspondances entre Escher et Perec
}

\section{Daniela Tononi}

\section{(2) OpenEdition}

\section{Journals}

\section{Édition électronique}

URL : http://journals.openedition.org/rief/810

DOI : $10.4000 /$ rief. 810

ISSN : 2240-7456

\section{Éditeur}

Seminario di filologia francese

\section{Référence électronique}

Daniela Tononi, «Op art et roman virtuel : correspondances entre Escher et Perec », Revue italienne d'études françaises [En ligne], 2 | 2012, mis en ligne le 15 décembre 2012, consulté le 01 mai 2019. URL : http://journals.openedition.org/rief/810 ; DOI : 10.4000/rief.810

Ce document a été généré automatiquement le 1 mai 2019.

\section{(c) (i) (9)}

Les contenus de la RIEF sont mis à disposition selon les termes de la Licence Creative Commons Attribution - Pas d'Utilisation Commerciale - Pas de Modification 4.0 International. 


\title{
Op art et roman virtuel : correspondances entre Escher et Perec
}

\author{
Daniela Tononi
}

\subsection{Introduction : abstraction versus figuration}

Dans l'évolution complexe de l'art abstrait qui caractérise la seconde moitié du $\mathrm{XX}^{\mathrm{e}}$ siècle, Escher (1898-1972) semble presque absent : bien qu'il reste à l'écart des bouleversements contemporains, il propose un art qui, d'une part, révèle l'influence de l'abstraction et, d'autre part, anticipe les illusions de l'op Art. Il partage avec l'art abstrait l'abandon de l'imitation du réel en lui substituant la représentation d'un «irréel logique » conçu en tant que «jardin magnifique [...] dont la porte reste grande ouverte à tous $»^{1}$ car il est possible de retrouver une explication logique même aux images impossibles et de découvrir finalement l'œuvre comme une révélation.

2 Après une période caractérisée par la réalisation des paysages du Sud de l'Italie, à partir de 1937 Escher se consacre à l'expérimentation qui porte sur la structure de l'espace et de la surface plane. Ainsi Bruno Ernst distingue trois thèmes qui caractérisent la production eschérienne ${ }^{2}$ : «la structure de l'espace» qui est interrogée dans les estampes de paysages, par l'interpénétration de mondes divers et dans la réalisation de solides mathématiques abstraits; «la structure de la surface plane » dont l'étude permet de réaliser les métamorphoses ${ }^{3}$, les circuits fermés ${ }^{4}$ et les approches de l'infini ${ }^{5}$. Enfin, en ce qui concerne la production qu'Ernst indique comme « relation entre l'espace et la surface plane par rapport à la représentation ", Escher propose de nouvelles approches de la perspective qui permettent la représentation d'images impossibles.

Les structures mathématiques, la notion de réalité comme « merveilleuse, et au fond, très humaine illusion $»^{6}$, la structure énigmatique, qui donne aux œuvres eschériennes la même structure que celle d'un roman policier, et le défi à l'infini permettent d'établir des convergences significatives entre le graveur hollandais et Georges Perec, bien que dans 
son œuvre les allusions explicites à Escher soient limitées au profit d'un Klee et d'un Magritte.

4 En effet, la peinture est un élément nécessaire et toujours présent dans les œuvres de Perec, à la fois dans son éventail citationnel et en tant qu'élément qui informe, d'une façon spéculaire, sur le fonctionnement structurel du roman. Ces deux fonctions, qu'il développe grâce aux références aux œuvres d'art, sont exploitées par exemple dans La vie mode d'emploi, dans Un cabinet d'amateur ou, encore, dans Un homme qui dort où la référence à Magritte sert de contrepoint à l'histoire d'un homme observé dans un état semiconscient. Mais dans ses romans l'art n'est pas seulement soumis à un procédé de fictionnalisation: il devient un objet de réflexion, un outil pour renouveler l'écriture. Ainsi dans sa Défense de Klee (1959), Georges Perec propose une lecture tout à fait intéressante du débat contemporain qui oppose l'abstraction à la figuration. S'opposant à la critique artistique contemporaine, il relit l'opposition en termes de distinction entre formalisme et « œuvre à contenu ». Perec définit ainsi formaliste « toute œuvre qui ne nous propose qu'une certaine "harmonie" (figurative ou non...), harmonie qui peut d'ailleurs être "dissonante" et se situer à des niveaux divers (plastiques, rythmiques, etc... ) $»^{7}$, tandis que l'œuvre à contenu permet d'activer chez le spectateur une émotion qui n'est pas un réflexe mais «le point de départ d'une réaction (démarche, prise de conscience, bonheur, euphorie dynamique, compréhension, prise de possession du monde, résolution de contradictions) $»^{8}$. Dans cette nouvelle approche du débat, Perec remarque surtout l'ambition de l'art non-figuratif qui veut « rendre compte du monde sans passer par sa représentation $»^{9}$. L'acception négative donnée au formalisme au profit d'une plus grande ambition de «l'œuvre à contenu» permet de déplacer le débat concernant l'abstraction des techniques aux résultats : une œuvre est plus intéressante si elle permet une réception active. Le refus de l'acceptation passive de l'œuvre d'art que Perec transpose à ses romans devient un postulat dans l'œuvre d'Escher, qui peut alors être inclus parmi les œuvres que Perec appelle « à contenu ».

\subsection{La manipulation de l'espace}

Dans l'œuvre de Georges Perec, on ne trouve que de très rares allusions explicites à Escher et à ses gravures. Ainsi dans La boutique obscure (1973), recueil de 124 récits de rêves, le rêve 41 propose une réélaboration onirique de la lithographie Montée et descente (1960) d'Escher :

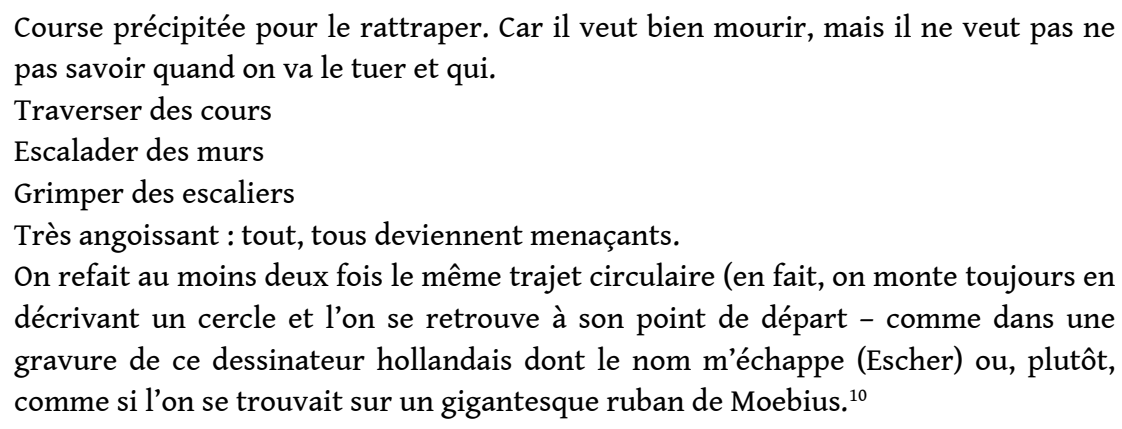

6 La lithographie d'Escher à laquelle Perec fait allusion représente l'une des tentatives d'approche de l'infini : si l'on suit l'un des personnages qui se trouvent sur l'escalier de la lithographie, on a l'impression qu'il monte ou descend, bien qu'il reste toujours sur le même plan parce qu'il est impossible de distinguer le haut et le bas. Cette illusion optique 
est créée par l'association géométrique du plan horizontal sur lequel se trouve l'escalier et de la spirale sur laquelle sont disposés les autres éléments du bâtiment.

7 Mais dans le texte de Perec, l'allusion à Escher fonctionne en tant que traduction visuelle de l'angoisse et de "l'inquiétante étrangeté » que dans les rêves Freud attribue «au retour du semblable ", « au retour involontaire au même point $»^{11}$. Le texte de Perec, où l'image eschérienne et le ruban de Moebius synthétisent le sens du mouvement perpétuel qui devient menaçant dans sa répétition, anticipe l'un des éléments qui permet de rapprocher l'écrivain du graveur: une nouvelle interprétation de l'espace qui contredit toute loi rationnelle préconstituée. Ainsi les mondes impossibles d'Escher ou les tentatives de représenter l'infini dans l'espace limité de la feuille et les romans de Perec avec leurs proliférations diégétiques suggèrent une nouvelle façon de percevoir la réalité.

8 La mise en crise de toute idée reçue concernant l'espace et ses lois dans Espèces d'espaces de Perec nie toute possibilité d'une reconstruction réelle : « ...l'espace n'existe pas » dit-il, "[...] L'espace, c'est ce que je peux en transcrire, et ce que j'en transcris est nécessairement faux $»^{12}$.

Pour cette raison, Perec substitue à la fidèle reproduction de l'espace « quelque chose qui est $d u$ domaine $d u$ construit mais qui est une construction mentale $»^{13}$. Cette réinterprétation de la notion d'espace permet de rapprocher la démarche perecquienne de celle d'Escher, qui propose dans son œuvre la construction mentale d'un irréel logique. Ainsi les illusions optiques qui caractérisent les tableaux eschériens peuvent être considérées comme l'application en peinture du même procédé d'abstraction appliqué aux espaces géométrique et architectural.

10 C'est dans le prière d'insérer d'Espèces d'espaces ${ }^{14}$ que Perec remarque la nécessité d'« interroger» l'espace pour répondre au sens de confusion provoqué par les « fissures », les « hiatus », les " points de friction » qui caractérisent l'espace de notre vie. Cette œuvre ${ }^{15}$ parcourt ainsi le concept d'espace depuis son acception matérielle de page conçue comme le simulacre d'espace jusqu'à son abstraction en tant que représentation littéraire et picturale. Cette expansion progressive qui se développe par encerclements successifs restitue sa conception de l'espace qui ressemble «à un oignon avec des sphères successives $»^{16}$ :

Et comme cet espace était au départ un espace d'écriture, on commence par la page blanche et, à partir de ce moment-là, une fois qu'on a commencé à sillonner l'espace, puisque quand on fait ça on fait des sillons, le livre s'en va, en essayant de s'élargir : comme un caillou qu'on jette dans une rivière et qui fait des cercles. ${ }^{17}$

11 Cette idée "presque cosmologique » de l'espace trouve sa correspondance visuelle dans Sentier de vie II du graveur hollandais : il s'agit d'une tentative d'approche de l'infini où l'image est construite par deux spirales logarithmiques qui se produisent à partir de deux points situés sur une circonférence. 


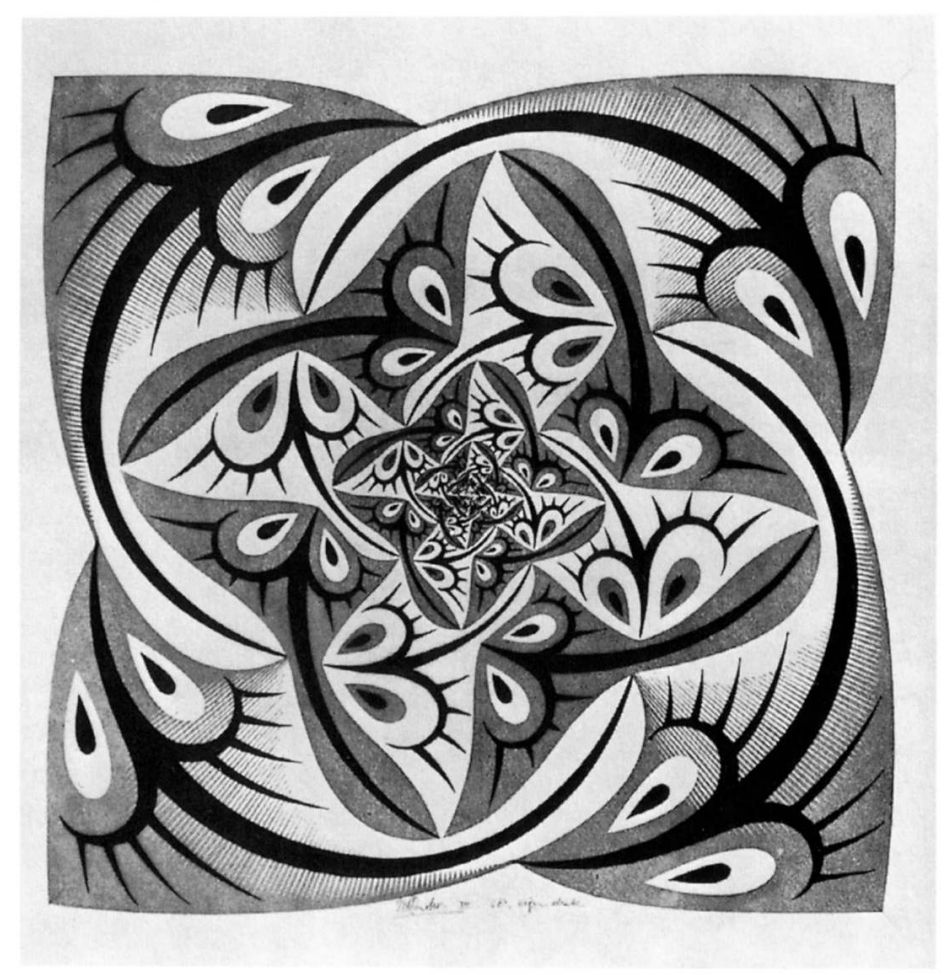

Maurits Cornelis Escher, Sentier de vie II

Image tirée de Bruno Ernst, Le miroir magique de M.C. Escher, (Köln, Taschen, 1994) mort par la représentation du cycle vital des poissons qui naissent petits au centre de l'œuvre et qui grandissent jusqu'à s'abîmer et redevenir petits et infinis au centre de l'image. Ce défi lancé à l'espace limité de la feuille pour tenter de représenter l'infini répond aussi à l'une des expérimentations géométriques d'Escher: le remplissage périodique du plan ${ }^{18}$ qui constitue une variante systématique de l'art du puzzle.

Tandis qu'Escher essaye de conquérir l'espace de la feuille en surmontant ses limites matérielles par l'illusion optique, dans l'œuvre de Perec la conquête de l'espace se réalise par degrés en considérant les espaces de la quotidienneté dans lesquels nous vivons et qui rythment le temps de notre journée, car «Vivre, c'est passer d'un espace à un autre, en essayant le plus possible de ne pas se cogner $»^{19}$. Mais le regard porté sur les espaces, même les plus banals (le lit, la chambre, l'appartement), est soumis à un phénomène d'association qui produit deux sortes d'imagination, à savoir l'imagination passive qui se traduit par les souvenirs et l'imagination active: «L'espace ressuscité de la chambre suffit à ranimer, à ramener, à raviver les souvenirs les plus fugaces, les plus anodins comme les plus essentiels. [...] C'est sans doute parce que l'espace de la chambre fonctionne chez moi comme une madeleine proustienne $»^{20}$. L'expansion progressive de l'espace considéré coïncide avec une raréfaction du souvenir personnel au profit de l'imagination ou des limites de l'imagination. Si la page, le lit et la chambre fonctionnent en tant qu'espaces individuels, l'appartement, l'immeuble, la rue, le quartier, la ville, la campagne et le pays activent en tant que lieux pluriels une autre typologie d'intervention, pas forcément rationnelle. Si Perec essaye d'imaginer un appartement avec une chambre sans fonction, qu'il appelle «espace inutile », et si encore l'attention 
portée sur l'immeuble se concrétise dans le projet de La vie mode d'emploi, la dernière partie d'Espèces d'espaces développe le thème d'espace à partir du concept de perspective :

Nous nous servons de nos yeux pour voir. Notre champ visuel nous dévoile un espace limité : quelque chose de vaguement rond, qui s'arrête très vite à gauche et à droite, et qui ne descend ni ne monte bien haut. [...] Notre regard parcourt l'espace et nous donne l'illusion du relief et de la distance : c'est ainsi que nous construisons l'espace: avec un haut et un bas, une gauche et une droite, un devant et un derrière, un près et un loin. ${ }^{21}$

C'est l'impuissance à saisir l'espace visible qui contraint l'observateur à substituer à la simple contemplation des actions qui lui permettent une activité compensatoire. Ainsi pour Escher et Perec deux actions sont possibles avec l'espace : jouer et le conquérir.

Pour Perec l'espace, loin de suggérer l'idée d'infini, devient « ce qui arrête le regard, ce sur quoi la vue bute : l'obstacle [...] $»^{22}$ et, donc, un objet à manipuler. La conquête de l'espace n'est pas alors le résultat d'un déplacement réel, mais une conquête qui se réalise grâce à l'écriture et par la manipulation ludique des distances, des mesures et des lois qui règlent l'espace. Ainsi, parmi les exemples ludiques proposés, Perec remarque: "Commencer à s'habituer à vivre en état d'apesanteur: oublier les verticales et les horizontales: gravures d'Escher, l'intérieur des véhicules interplanétaires dans 2001, Odyssée dans l'espace $»^{23}$.

16 Cette référence à Escher est une allusion à la lithographie Relativité, qui propose la coexistence de trois mondes différents et, en général, aux techniques du graveur hollandais qui se caractérisent par la manipulation du concept d'espace. Ainsi, lorsque Perec cherche à établir la fonction des pièces d'un appartement, essayant d'imaginer un espace sans fonction, « Non pas "sans fonction précise", mais précisément sans fonction; non pas pluri-fonctionnel (cela, tout le monde sait le faire), mais a-fonctionnel $»^{24}$, ce sont les gravures d'Escher, L'immortel de Borges et Magritte qui lui donnent la possibilité de concevoir, au moins, l'inutile :

Comment penser le rien? Comment penser le rien sans automatiquement mettre quelque chose autour de ce rien, ce qui en fait un trou, dans lequel on va s'empresser de mettre quelque chose, une pratique, une fonction, un destin, un regard, un besoin, un manque, un surplus ? J'ai essayé de suivre avec docilité cette idée molle. J'ai rencontré beaucoup d'espaces inutilisés. Mais je ne voulais ni de l'inutilisable, ni de l'inutilisé, mais de l'inutile. [...] j'ai pensé à une nouvelle de Borges (L'Immortel) [...] j'ai pensé à des gravures d'Escher et à des tableaux de Magritte [.....2.

17 Cette aspiration à l'inutile, à penser le rien, coïncide, comme le remarque RoparsWilleumier ${ }^{26}$, avec l'un des mouvements qui caractérise le texte perecquien où l'écriture établit un ordre textuel presque géométrique, permet de maîtriser l'espace de la page grâce aux longues descriptions, aux listes et aux inventaires.

18 La tension entre l'impuissance à maîtriser l'espace réel et l'ordre auquel l'espace est soumis par l'écriture aboutit dans l'œuvre de Perec à l'infra-ordinaire, à savoir «le contraire de l'événement ${ }^{27}$, "le bruit de fond que constitue chaque instant de notre quotidienneté $»^{28}$. Cette tension atteint, dans l'œuvre d'Escher, l'inclusion de l'impossible dans le possible, de l'irréel dans le réel. Si Perec, proche des peintres hyperréalistes américains, aboutit au fantastique et à l'imaginaire par une accumulation des descriptions et des objets, Escher arrive à rendre l'extraordinaire au plan du réel :

Il me semble parfois que nous souffrons du désir irrépressible de saisir l'impossible.

La réalité, le monde tridimensionnel qui nous entoure semble à nos yeux trop banal, trop ennuyant, trop ordinaire. Nous avons envie d'anormal ou de surnaturel, 
de ce qui n'existe pas, d'un miracle. Comme si la réalité quotidienne n'était pas assez mystérieuse ! [...] Mais il arrive que nous devenions réceptifs à l'inexplicable, à ce miracle dans lequel nous vivons constamment. ${ }^{29}$

\subsection{Infini ...} d'Escher il est possible de distinguer différentes typologies de pavage qui découlent de la forme des pièces composantes. Mais la classification proposée par Odifreddi tient aussi en compte la relation mutuelle des pavés qui peuvent donner un pavage isoédrique s'ils ont la même fonction, ou se combiner en suivant une seule façon de recouvrir le plan s'ils ne sont pas congruents. Cette classification répond aux typologies relevées par Perec lors d'un entretien sur l'art du puzzle dans La vie mode d'emploi. Perec appelle «puzzle trivial ", le faux puzzle composé de pièces "toutes découpées de la même manière, pratiquement superposables", tandis que le vrai puzzle se compose de "pièces minuscules et de formes extrêmement variées $»^{31}$. Mais le puzzle acquiert dans l'œuvre de Perec une double fonction, car dans La vie mode d'emploi, le puzzle est l'image qui pousse le développement diégétique aussi bien que la contrainte qui fonde la structure du roman: «Il y avait cette image de puzzle qui était tout le temps en train, comme si le livre était lui-même un puzzle et les pièces de la maison des chapitres devant se relier les uns aux autres même si on ne voyait pas, chaque fois, exactement comment cela se combinait $»^{32}$.

Mais le puzzle devient aussi métaphore du processus interprétatif qui se fonde sur la dichotomie vrai/faux, comme le remarque Perec à propos des histoires qui composent $L a$ vie mode d'emploi :

[...] qu'est-ce qui est vrai ? Et qu'est-ce qui est faux ? Parce qu'il y a tout un jeu. C'est un livre avec lequel on joue, je crois comme on joue avec le puzzle : c'est pour cette raison qu'il a un index à la fin. Pour que l'on puisse reconstituer soi-même des histoires ou suivre des histoires qui ne sont pas entièrement racontées. ${ }^{33}$

21 Ainsi Perec, dans le préambule, remarquant le rapport nécessaire entre la pièce du puzzle et l'ensemble car « seule compte la possibilité de relier cette pièce à d'autres pièces », fait allusion à la structure de son roman :

L'image centrale du livre est un puzzle. Au-delà, il y a l'image d'une œuvre [...] où tout ce que je vais écrire s'organisera à son tour comme un puzzle dont chaque livre serait l'une des pièces. L'ensemble de tout cela participera au puzzle de la littérature contemporaine dont les pièces sont les écrits des autres auteurs. Et moi, je vais remplir un vide dans ce puzzle. Ce que j'aime dans l'idée du puzzle [...] c'est qu'une pièce isolée n'a pas de sens en soi. Elle est inerte. C'est la capacité de la relier aux autres qui lui donne son sens sa cohérence. Une fois qu'on a reconstitué l'image, on a fini le jeu, il ne reste plus rien. ${ }^{34}$

Si, dans les gravures d'Escher, c'est le pavage euclidien du plan qui permet de créer l'illusion de l'infini en tant que forme de répétition du semblable, dans l'œuvre perecquienne c'est le puzzle qui fait « proliférer à l'infini » ${ }^{35}$ le roman et le transforme en œuvre potentielle.

Mais le puzzle ou le pavage ne sont pas la seule forme de réduplication : dans Un cabinet d'amateur de Perec et dans Exposition d'estampes d'Escher, c'est la mise en abyme qui permet d'atteindre l'infini fictionnel ou visuel. Dans son essai Le récit spéculaire, Dällenbach remarque que la mise en abyme peut avoir trois variantes qui découlent des degrés d'analogie entre «la mise en abyme de l'énoncé et l'objet qu'elle réfléchit » ${ }^{36}$. Les 
trois formes de mise en abyme se réalisent d'après trois principes différents : la similitude, le mimétisme et l'identité. Ainsi, Dällenbach appelle « dédoublement simple » le procédé qui par similitude réitère " une même œuvre ", tandis que le "dédoublement à l'infini » permet la réduplication de « la même œuvre » en relation mimétique avec l'objet qu'elle réfléchit. Au principe d'identité, enfin, le critique associe le « dédoublement paradoxal » qui permet de réfléchir « l'œuvre même ».

Exemple de dédoublement paradoxal, Un cabinet d'amateur, « délicieusement agaçant à la manière des dessins d'Escher $\aleph^{37}$, est un récit fort complexe, d'une part parce qu'il est " une miniaturisation et une copie altérée et déguisée de La Vie mode d'emploi » ${ }^{38}$, d'autre part parce que la mise en abyme, qui se concrétise par l'inclusion au niveau diégétique de l'œuvre homonyme du peintre Heinrich Kürz, se pose en relation spéculaire et métatextuelle avec la structure même du roman perecquien. Ainsi la description minutieuse de la toile du peintre Kürz, qui rassemble plus de cent tableaux, module en même temps les variations entre les différentes copies. La réduction infinie de « tous ces tableaux à nouveau reproduits, et ainsi de suite sans rien perdre de leur précision dans la première, dans la seconde, dans la troisième réflexion jusqu'à n'être plus sur la toile que d'infimes traces de pinceaux $\|^{39}$ transforme en paradoxe le concept d'originalité de l'œuvre d'art en proposant donc des copies modifiées, qui ne sont pas identiques entre elles et qui finalement se révèlent des copies de faux. Ainsi, le vertige diégétique entre vrai et faux aboutit aussi à la rupture de l'illusion fictionnelle: "Des vérifications entreprises avec diligence ne tardèrent pas à démontrer qu'en effet la plupart des tableaux de la collection Raffke étaient faux, comme sont faux la plupart des détails de ce récit fictif, conçu pour le seul plaisir, et le seul frisson, du faire-semblant $»^{40}$.

Si dans le texte perecquien la mise en abyme fonctionne par concentration et par réflexion, car elle inclut dans la toile de Kürz des tableaux de plus en plus petits, en 
revanche dans l'Exposition d'estampes d'Escher la construction géométrique de la mise en abyme se développe par dilatation.

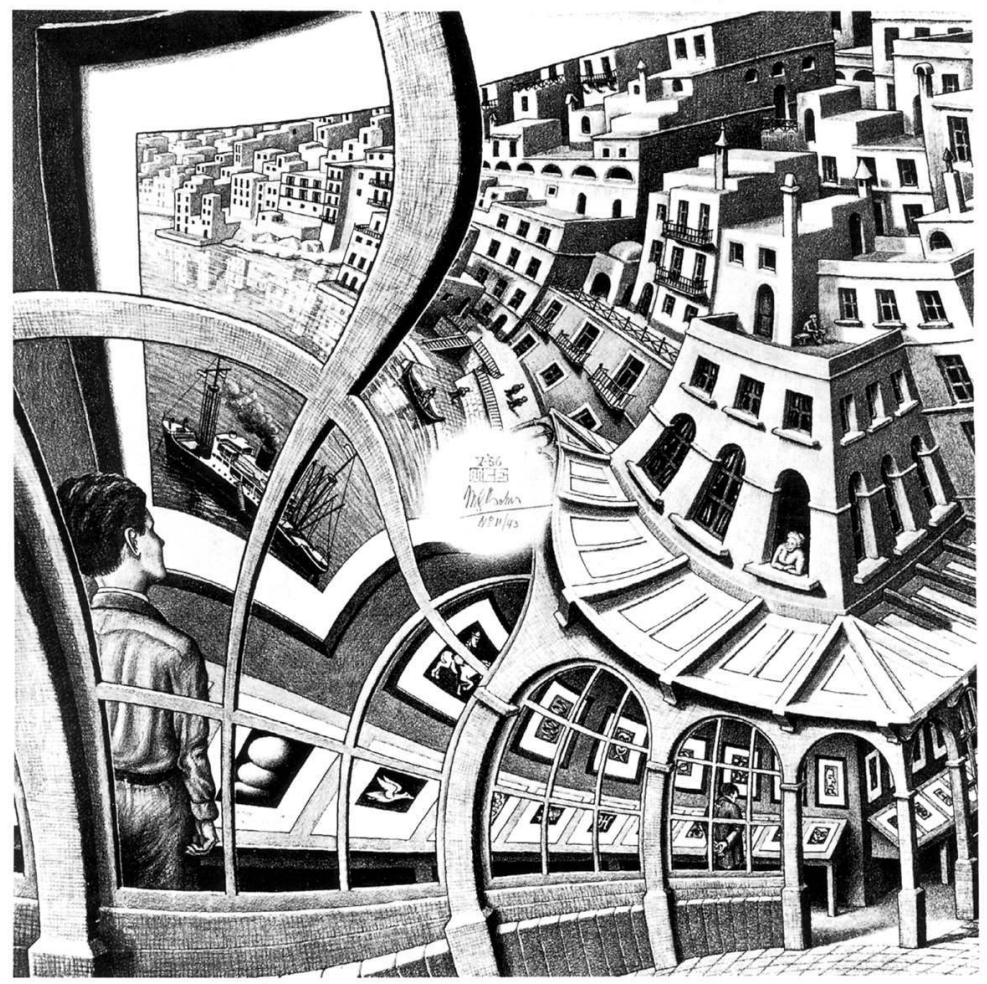

Maurits Cornelis Escher, Exposition d'estampes

Image tirée de Bruno Ernst, Le miroir magique de M.C. Escher, (Köln, Taschen, 1994)

La figure originale de cette lithographie a été soumise à plusieurs stades d'agrandissement car, comme le remarque Bruno Ernst, « en partant de la droite en bas vers la gauche en haut, nous voyons la galerie s'agrandir $»^{41}$. L'œuvre représente une extraordinaire variante de la mise en abyme car elle inclut en même temps l'auteur grâce au rectangle avec la signature d'Escher au centre de l'image et du spectateur. Comme le remarque Bernard Guy, ce dessin qui « est à la fois un tableau que l'on contemple et la ville qui contient l'exposition $»^{42}$, altère le rapport spatio-temporel car il propose une fausse continuité spatiale et une discontinuité temporelle. La variabilité des gravures d'Escher découle alors de la place prise par le récepteur: le déplacement dans la contemplation de l'œuvre ne provoque pas seulement un changement de perspective mais, démentit en même temps la première vision.

Ainsi, si dans la toile de Kürz l'illusion de l'infini est restituée par l'inclusion concentrique des «répétitions de plus en plus minuscules ${ }^{43}$, dans la lithographie d'Escher la «dilatation fermée en forme d'anneau» qui constitue l'armature de l'œuvre n'a ni commencement, ni fin.

\subsection{Conclusion}

Les œuvres réalisées par Escher après 1937 abandonnent la représentation des choses pour lui substituer l'expression d'une « vision intérieure » du monde. À la différence des peintres surréalistes qui traduisent dans leur œuvre un irréel qui n'exige pas l'application d'une herméneutique logique, Escher, même avec ses «mondes impossibles ", sollicite 
toujours l'intervention de l'intellect en superposant à son irréel figuratif une logique graphique que «seuls ceux qui sont prêts à aller au-delà des apparences $»^{44}$ peuvent comprendre. Ce caractère ludique, qui active une autre modalité de réception de l'œuvre, qui n'est plus conçue comme contemplation passive, coïncide avec le processus interprétatif que Perec demande à son lecteur. Aux illusions optiques des gravures d'Escher, Perec substitue l'infinie prolifération du roman qui devient virtuel parce qu'il accueille « une histoire et l'histoire de cette histoire, une fiction et une fiction ayant trait à la fiction, comme un jeu de miroirs. Et ça ne se termine pas avec la fiction et la fiction relative à la fiction : il y aura une spéculation portant sur la fiction relative à la fiction et ainsi de suite $[. ..] »^{45}$.

\section{NOTES}

1. M. C. Escher, MC Escher on Escher : Exploring the Infinite, New York, Harry N. Abrams, 1989, p. 93.

2. B. Ernst, Le miroir magique de M. C. Escher, tr. fr. J. Renault, Köln, Taschen, 1994, p. 24-25.

3. Ibid., p. 41 : «Dans la métamorphose, nous voyons comment les formes vagues et abstraites se convertissent en formes concrètes et bien délimitées, et vice versa ».

4. Les circuits fermés s'obtiennent par le remplissage périodique d'un plan où la phase terminale devient la phase initiale.

5. M. C. Escher, MC Escher on Escher : Exploring the Infinite, cit., p. 93 : «Un plan, qu'il faut imaginer comme une surface s'étendant dans toutes les directions, sans frontières, peut être rempli ou divisé à l'infini, selon un certain nombre de systèmes, au moyen de figures géométriques semblables, qui se touchent l'une l'autre de tous les côtés et ne laissent aucun "vide" ».

6. J. L. Locher, M. C. Escher, New York, H. N. Abrams, 1992, p. 68.

7. G. Perec, "Défense de Klee ", dans Cahiers Georges Perec-L'œil d'abord... Georges Perec et la peinture, n.6, Paris, Seuil, 1996, p. 16-26, p. 17.

8. Ibid., p. $18:$ : [...] quant à l'œuvre à contenu [...] on peut toujours essayer de la définir en disant que la chose représentée n'est que le support sensible d'une émotion demandée au spectateur ».

9. Ibidem.

10. G. Perec, La boutique obscure, 124 rêves, Paris, Denoël, 1973.

11. S. Freud, "L'inquiétante étrangeté », dans Essais de psychanalyse appliquée, Paris, Gallimard, 1971, p. 163-211, p. 188.

12. G. Perec, «À propos de la description », dans D. Bertelli et M. Ribière (éd.), Perec. Entretiens et conférences 1979-1981, vol. II, Nantes, Joseph K., 2003, p. 227-243, p. 240.

13. Ibidem.

14. G. Perec, Espèces d'espaces, Paris, Galilée, 2000.

15. Perec remarque (Ibid., p. 13) : «L'objet de ce livre n'est pas exactement le vide, ce serait plutôt ce qu'il y a autour, ou dedans. Mais enfin, au départ, il n'y a pas grand-chose : du rien, de l'impalpable, du pratiquement immatériel [...]».

16. Perec résume ainsi la construction de son œuvre: «Ma première approximation d'espace, c'était la page ; après la page, on va commencer des emboîtements avec un jeu de mots sur "la page" et "le page", c'est-à-dire, en argot, le lit. Puis, du lit, on va passer à la chambre, de la chambre à l'appartement, de l'appartement à la maison, de la maison à la rue » («Entretien 
Georges Perec/Ewa Pawlikowska », dans D. Bertelli et M. Ribière (éd.), Perec. Entretiens et conférences 1979-1981, cit., p. 199-207, p. 203).

17. Ibid., p. 204.

18. L'étude d'une logique systématique naît de la fascination lors d'une visite à l'Alhambra : « En voyant les murs et les planchers couverts de tuiles aux couleurs vives des palais de l'Alhambra, en Espagne, on comprend que les Maures étaient passés maîtres dans l'art de remplir un plan par des figures semblables, imbriquées, qui se touchent sans laisser de vides. Les artistes japonais ont également produit d'excellents exemples de ces curieux motifs. Quel dommage que leur religion ait interdit aux Maures de faire des images!» (M. C. Escher, MC Escher on Escher: Exploring the Infinite, cit., p. 25).

19. G. Perec, Espèces d'espaces, cit., p. 16.

20. En faisant allusion au projet de faire l'inventaire de tous les lieux où il a dormi, Perec remarque: «ce projet [...] ne voudrait rien être d'autre que le strict développement des paragraphes 6 et 7 du premier chapitre de la première partie (Combray) du premier volume (Du côté de chez Swann) de À la recherche du temps perdu » (Espèces d'espace, cit., p. 47).

21. Ibid., p. 159.

22. Ibidem.

23. Ibid., p. 168.

24. Ibid., p. 67.

25. Ibid., p. 67-69.

26. Voir Ropars-Wuilleumier, Écrire l'espace, Saint Denis, Presses Universitaires de Vincennes, 2002, p. 25.

27. G. Perec, « Entretien avec Gérard Dupuy », dans D. Bertelli et M. Ribière (éd.), Perec. Entretiens et conférences 1965-1978, I, cit., p. 232-235, p. 234.

28. G. Perec, « Entretien avec Jean-Marie Le Sidaner », dans L'Arc, 76, 1979, p. 3-10, p. 4.

29. M. C. Escher, MC Escher on Escher : Exploring the Infinite, cit., p. 135.

30. P. Odifreddi, « Maurits Cornelis Escher : l'arte del puzzle », dans Le Scienze, 340, Milano, 1996, p. 42-49.

31. G. Perec, «La vie: règle du jeu », A. Hervé (propos recueillis par), dans D. Bertelli et M. Ribière (éd.), Perec. Entretiens et conférences 1965-1978, cit., p. 267-285, p. 268.

32. G. Perec, Entretien (avec Gabriel Simony), Paris, Le Castor Astral, 1989, p. 9-10.

33. Ibid., p. 11.

34. G. Perec, « La vie : règle du jeu », cit., p. 268.

35. G. Perec, «Come ha fatto a costruire quel "puzzle infernale" ?» E. Guicciardi, (propos recueillis par) tr. fr. D. Bertelli, dans D. Bertelli et M. Ribière (éd.), Perec. Entretiens et conférences 1965-1978, cit., p. 261.

36. L. Dällenbach, Le récit spéculaire. Essai sur la mise en abyme, Paris, Seuil, 1977, p. 142.

37. A. Chauvin, «Le jeu des erreurs ou métamorphoses en minuscules», dans Études littéraires, vol. XXIII, 1-2, 1990, p. 87-110, p. 91.

38. Ibid., p. 100.

39. G. Perec, Un cabinet d'amateur, dans Georges Perec. Romans et récits, Paris, Le livre de poche, « La Pochothèque ", 2002, p. 1378.

40. Ibid., p. 1421.

41. B. Ernst, Le miroir magique de M. C. Escher, cit., p. 37.

42. B. Guy, L'éclair et le tonnerre, promenades entre l'espace et le temps: à propos de la théorie de la relativité, Paris, Éditions Publibook, 2004, p. 167.

43. G. Perec, Un cabinet d'amateur, cit., p. 1378.

44. M. C. Escher, MC Escher on Escher : Exploring the Infinite, cit., p. 136.

45. G. Perec, «La fiction et son faire », dans D. Bertelli et M. Ribière (éd.), Perec. Entretiens et conférences 1979-1981, cit., p. 251-260, p. 257. 
INDEX

Mots-clés : Perec (Georges), Escher, espace, puzzle, abstraction, figuration, mise en abyme, Op Art 